\title{
Experimental Study on Load-Displacement Relationship of Prestressed Cable in Red Clay Stratum
}

\author{
Yong Xing JI ${ }^{1,2, a}$, Yuan Jie XIANG ${ }^{1,2, b}$, Xiao Yong ZHAO ${ }^{1,2, c}$ \\ ${ }^{1}$ Guizhou Construction Science Research and Design Institute of CSCEC, Guiyang 550000, China. \\ ${ }^{2}$ Inspection Centre of Construction Science Research of Guizhou Province, Guiyang 550000, China.
}

\begin{abstract}
The P-S curves of prestressed anchor cable are obtained by field pull-out tests in the red-clay stratum in Guiyang area. The P-S curves of different theoretical models are discussed base on the theoretical analysis. The elastic and plastic displacement of prestressed anchor cable are analyzed. The results shows that, the anchoring effect are mainly effected by the red-clay mechanic properties in the red-clay stratum; the P-S curve of anchor bolt can be fitted well by the exponential model and conformed to the actual; the plastic displacement is used to anchor's damage index are more truthfulness in the red-clay stratum.
\end{abstract}

\section{Introduction}

Prestressed anchors as a safe, reliable and cost-effective geotechnical reinforcement measures have been widely used in slope reinforcement, dam engineering, foundation pit engineering and tunnel and underground chamber engineering, the outer anchorage section provides tension, Section anchors and anchors and anchors and rock mass interface will be transmitted to the rock mass ${ }^{[1]}$. The failure modes of the prestressed anchor are mainly in the following forms: the cable strand breaks; the slippage between the strand and the anchoring agent; the slippage between the anchoring agent and the wall of the hole; the anchored rock Weakened; between the strand and the hole between the anchoring agent weakened. Engineering practice, the third form of the most common destruction ${ }^{[2]}$.

The research on the strengthening mechanism of the prestressed anchor cable under different soil conditions is not deep, and the anisotropy of the rock and soil itself .It is very necessary to study the mechanism of prestressed anchor cable under certain soil conditions. Based on this, the ultimate drawing test of the prestressed anchor cable is carried out, and the comparative analysis and research on the ultimate pull out force and failure mechanism of the prestressed anchor cable in the red clay stratum of Guizhou are carried out.

\section{Prestressed Anchor Cable Load-Displacement Curve Model}

\subsection{Double-broken Line Model}

When the interface of the anchor layer is in the elastic phase, the relationship between load and displacement of the anchor cable under the pulling force is given by the following formula: ${ }^{[3]}$

$u=P\left[\frac{L_{1}}{E_{s} A_{s}}+\frac{4 \operatorname{Coth}\left(\beta L_{b}\right)}{\pi D^{2} E_{a} \beta}\right]$

When the anchoring interface is in the plastic stage, the relationship between the load and displacement of the anchor cable under the pulling force is given by the following formula:

$u=\frac{4 \tau_{1}}{\beta^{2} D E_{a}}-\frac{2 \tau_{2}}{D E_{a}} L_{P}^{2}+\frac{4 P}{\pi D^{2} E_{a}} L_{p}+\frac{P L_{1}}{A_{s} E_{s}}$

In the formula (2), $E_{a}$ is the elastic modulus of the anchor, $E_{s}$ is the elastic modulus of the anchor, $D$ is the diameter of the anchor, $L_{l}$ is the length of the free section, $L_{b}$ is the length of the anchorage section, $A_{s}$ is the cross-sectional area of the anchor, $\beta$ is a constant, $\tau_{l}$ is the ultimate resistance Shear strength, $\tau_{2}$ is the residual shear strength, $u$ is anchor head displacement.

\subsection{Exponential Model}

Using the displacement solution of the Boussinesp problem with the vertical force acting on the Semi-infinite boundary, the formula for deriving the axial force distribution of the anchor anchorage section is shown below. ${ }^{[4]}$

$$
P=P_{u}\left(1-e^{a S}\right)
$$

In the formula (3), $P_{u}$ is the ultimate bearing capacity of the Anchor Cable, $a$ is the settlement attenuation factor, $P_{u}$ and $a$ can be determined by analysis of the pull-out test data. 


\subsection{Hyperbolic Model}

Using the load transfer function method, the shear stress of the anchorage section is analyzed and solved, and the distribution curve of shear stress along the axial direction of the anchorage section is simulated. ${ }^{[4]}$

$$
P=\frac{S}{a S+b}
$$

In the formula (4), $P$ is the anchor load $(\mathrm{kN}), S$ is the anchor displacement $(\mathrm{mm}), a$ and $b$ are the fitting parameter.

\section{Prestressed Anchor Cable Field Test}

\subsection{Project Overview}

The anchor cable pull-out test is done at the xingyunjiadiancheng foundation pit project in Guiyang. The soil layer of the foundation pit is a red clay stratum in the south area, and the stratum is more stable and has no bad geological phenomenon. The tensile strength design value $f_{p y}=1320 \mathrm{~N} / \mathrm{mm}^{2}$ is used for the 1860 grade steel wire with the specification $\Phi 15.2$. The design value of the prestressed design is $100 \mathrm{kN}$ and the single cable anchor is $110 \mathrm{kN}$.

\subsection{Pull-out Test Process}

The maximum test load of the single test cable is $150 \mathrm{kN}$ (1.5 times the design value of the prestress), the cable tensioning step:

(1) the overall pre-tensioning, pulling the maximum test load of $10 \%$ to eliminate the inelastic deformation of the anchor cable;

(2) The cable was subjected to a seven-stage pull-out test. Each stage of the tensile load, respectively, take the maximum test load of $20 \%, 20 \%, 20 \%, 10 \%, 10 \%, 10 \%$ and $10 \%$.

(3) When the following requirements are met, the acceptance shall be judged:

After loading to the maximum value of the test load plan, the deformation is stable.

(1) The total displacement measured under the maximum test load should exceed $80 \%$ of the theoretical elastic elongation of the free length of the anchor under the load and less than the theoretical extension of the length of the free section of the anchor Long value.

(2) Under the last load of 1 minute to 10 minutes, the anchor head is stable and the displacement is less than 1 $\mathrm{mm}$. When not satisfied, the continuous load 60 minutes, bolt displacement should be less than $2 \mathrm{~mm}$.

(4) An attack in the cable test may be considered as damage when one of the following conditions occurs.

(1) Anchor head displacement does not converge, the anchor from the rock layer pull out the anchor or pull out from the anchor;

(2) The total displacement of the anchor head exceeds the design allowable value;

(3) The increase of the anchor head displacement in the post-level load of the soil anchor test is more than twice the displacement of the upper load.

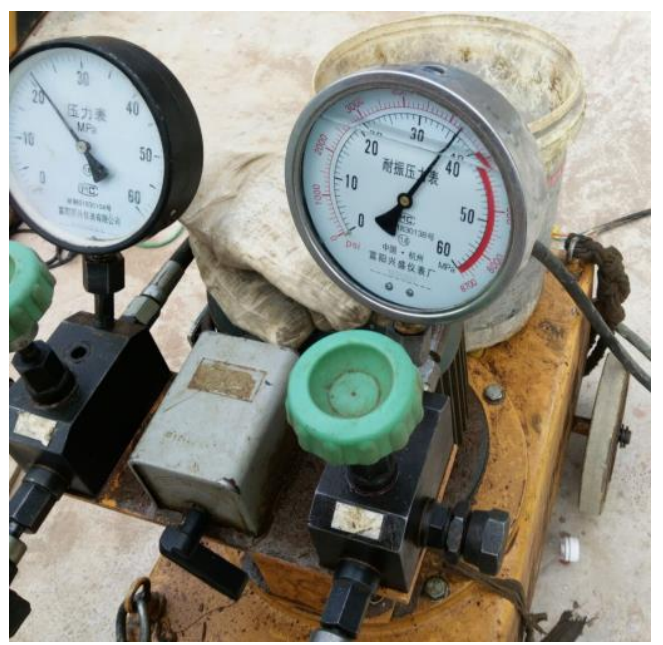

Fig.1 Manometer in the experiment

\section{Comparison of Test Results}

\subsection{Test to Determine the Results}

Field tests randomly selected 10 prestressed anchors in the site. According to the criteria described in 3.2, the test of the 10 anchor to determine the results shown in Table 1.

Tab.1 The testing results of the prestressed anchors

\begin{tabular}{lccccc}
\hline \multirow{2}{*}{ Specimen } & L $(\mathrm{m})$ & \multicolumn{2}{c}{ Anchor cable displacement $(\mathrm{mm})$} & \multirow{2}{*}{ Test result } \\
\cline { 3 - 5 } & & $\left(\Delta \mathrm{L}_{1}\right)$ & $\left(\Delta \mathrm{L}_{2}\right)$ & $(\Delta \mathrm{L})$ & \\
\hline $\mathrm{N} 13-2$ & 26.5 & 66.96 & 112.52 & 99.41 & Qualified \\
$\mathrm{N} 15-1$ & 33.5 & 113.07 & 163.51 & 143.35 & Qualified \\
$\mathrm{N} 18-1$ & 33.9 & 114.85 & 165.73 & 147.13 & Qualified \\
$2-10-1$ & 23.6 & 50.11 & 92.57 & 90.63 & Qualified \\
$2-13-2$ & 23.6 & 59.86 & 99.49 & 93.34 & Qualified \\
$2-15-1$ & 31.8 & 54.98 & 99.22 & 98.48 & Qualified \\
\hline
\end{tabular}




\begin{tabular}{cccccl}
\hline $2-17-1$ & 27.4 & 67.84 & 113.91 & 105.11 & Qualified \\
$2-18-1$ & 26.9 & 64.3 & 109.19 & 91.9 & Qualified \\
$2-19-1$ & 27.7 & 56.76 & 107.81 & 97.85 & Qualified \\
$2-20-1$ & 27.3 & 66.51 & 112.8 & 89.91 & Qualified \\
\hline
\end{tabular}

L- length of prestress anchor cable (m)

$\Delta \mathrm{L}_{1}-80 \%$ of the theoretical elongation value of the free-segment length.

$\Delta \mathrm{L}_{2}$ - The theoretical elongation value of the sum of the free-segment length and 1/2 anchorage length.

$\Delta \mathrm{L}$ - Maximum actual elongation of anchor under maximum experimental load.

Three of the test data were selected for processing and the $P-S$ curve was drawn, as shown in Figure 2.
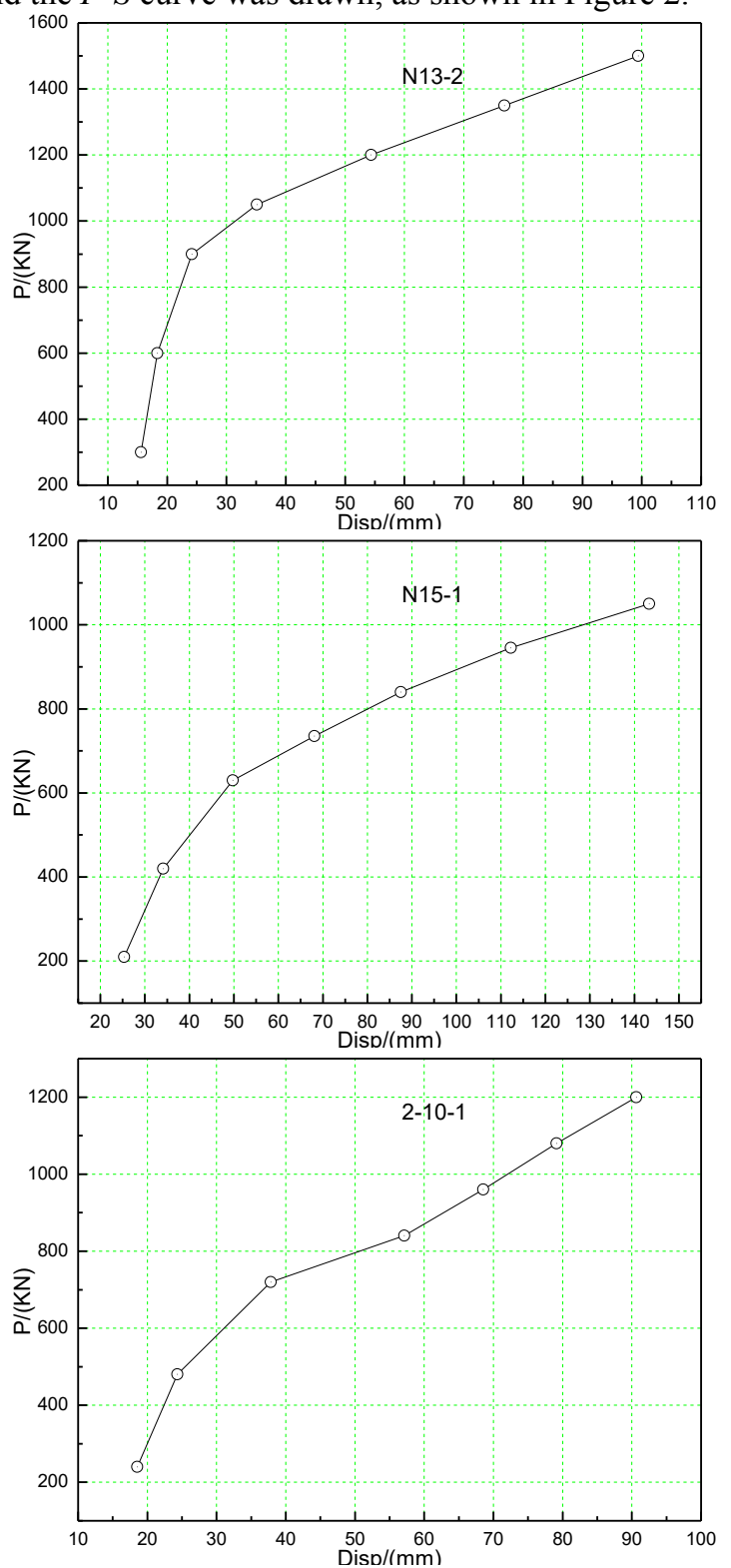

Fig. $2 P-S$ curves of the experiment

\subsection{Analysis of Anchoring Effect}

From the analysis of Fig. 2, the pull force and the displacement of the cable are basically linear. When the anchor displacement is small, the interface between the anchor and the anchor does not produce shear yield point, that is, the interface between the anchor and the anchor has not yet slipped or destroyed.

After the displacement of the anchor is obviously increased, the shear yield point is just generated at the interface between the anchor and the anchor, that is, the interface between the anchor and the anchoring agent is just sliding or breaking. After the displacement increases, the tension remains constant.

In the analysis of N13-2 in Fig. 2, when the pull force reaches $1000 \mathrm{KN}$ and the displacement reaches $35 \mathrm{rnm}$, the anchoring section of the anchor section is partially sheared and the $P-S$ curve is became flat.

When the displacement of the N15-1 cable reaches $700 \mathrm{KN}$ and the displacement reaches $55 \mathrm{~mm}$, the $P-S$ curve enters the plastic growth stage, which indicates that some shear failure occurs between the grout and the red clay.

Compared with the anchor cable number 2-10-1 and the anchor number N15-1 in Fig. 2, it can be seen that the displacement of the anchor cable increases with the increase of the pulling force in the elastic range, and the mechanical properties are the same.

The anchors 2-10-1 are pressure dispersible. When the anchorage length reaches a certain value, and then continue to increase the length of the anchor, can only lead to the potential of the cable material waste, while the load capacity to help little.

\subsection{P-S Curve Analysis}

According to the different load displacement theory model, the relevant parameters are substituted into the $P-S$ curve of each model, as shown in Fig.3

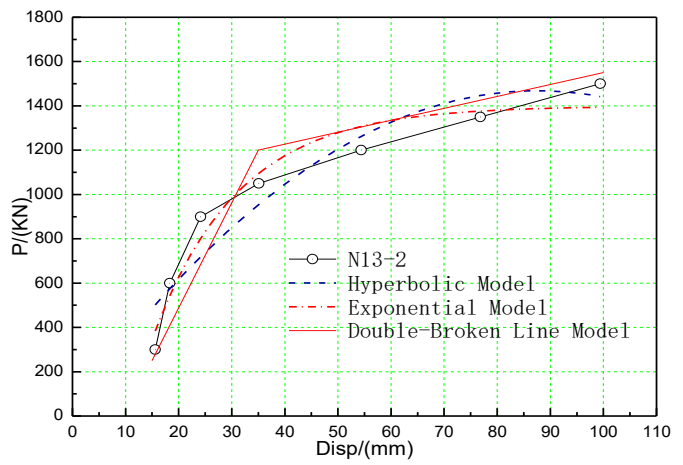



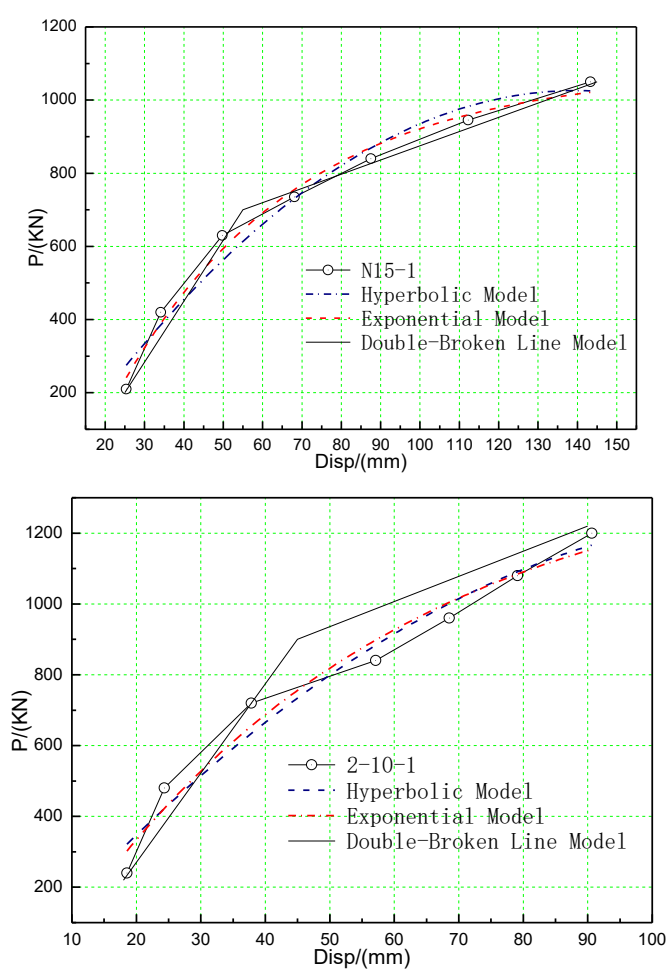

Fig.3 $P-S$ fitting curves of the experiment

From Figure 3 three kinds of prestressed anchor cable $P-S$ curve comparison shows that the displacement of the anchor head is not generated, and the distribution of the hyperbolic model and the exponential model is almost the same in the three cases.

In contrast, the accuracy of the fitting curve obtained by using the exponential model is high, and the obtained prediction bearing capacity is more reasonable, and the error between the measured values is very small.

Under the three cases, the prediction model of the ultimate bearing capacity of the anchor cable and the prediction accuracy of the double-broken line model at each stage load point are slightly worse.

Hyperbolic model and exponential model, the prediction accuracy of the load at all levels has a good fit with the measured $P-S$ curve. The accuracy of the load-displacement correlation error of the exponential model at each stage load is higher.

From the analysis of the $P-S$ curve with three kinds of anchor numbers, it is found that the damage form of the anchor cable in the red clay strata is mainly the relative displacement between the anchor and the soil when the anchor body has enough strength. That is, the plastic displacement, so the use of plastic in the red clay layer as the anchor of the destruction of the standard is more realistic.

The exponential model has better adaptability in the prediction of the ultimate bearing capacity of the red clay strata, and can be used as the index of prestressing control.

\section{Conclusion}

Through the pull-out test and the $P-S$ curve fitting model analysis of prestressed anchor cable in red clay stratum in Guiyang, the following conclusions can be drawn:

(1) In the red clay strata, the damage of the anchor body occurs mainly on the bonding surface of the slurry and the soil, and the anchor anchorage force is mainly affected by the mechanical properties of the red clay.

(3) Increasing the anchorage length of the anchor cable can not effectively improve the ultimate bearing capacity of the anchor cable. The anchoring length is not linearly related to the ultimate drawing force.

(3) In the red clay strata, the exponential model of the $P-S$ curve fitting of the prestressed anchor cable has better adaptability, which can provide the control standard for the prediction of the prestressing anchor cable performance index.

\section{Acknowledgments}

The authors gratefully acknowledge the support from Guizhou Provincial Science and Technology Department (QKH-SY[2015]3055).The authors also gratefully acknowledge the support provided through the research project of science and technology in China Construction Fourth Engineering Division Corporation Ltd (No. CSCEC4B-2015-KT-03).

\section{References}

1. You Chunan, Zhan Yubao. Distributing characters and analysis of stresses in prestressed cables. Chinese Journal of Rock Mechanics and engineering, 2005; 24(6):925-928

2. Fan Yujie, Zheng Qizhen, Wei Lin. Research on failure mechanism and ultimate load carrying capacity of prestressed cable. Chinese Journal of Rock Mechanics and engineering, 2005; 15:2765-2769

3. Li Jinhua, Yuan Bing. Research on relationship between anchor pulling capacity and displacement in soil. Coal Science and Technology, 2010;(03):32-34,38

4. Ying Zhimin, Zhang jie, Shang yuequan. Exponential model for simulating load-displacement curve of anchor rod.Rock and Soil Mechanics, 2005;(08):1331-1334 